\title{
21. PALEOMAGNETISM AND ROCK MAGNETIC PROPERTIES OF GABBRO FROM HOLE 894G, HESS DEEP ${ }^{1}$
}

\author{
Janet E. Pariso, ${ }^{2}$ Paul Kelso, ${ }^{3}$ and Carl Richter ${ }^{4}$
}

\begin{abstract}
Results of paleomagnetic and rock magnetic measurements are presented from gabbroic samples recovered during Ocean Drilling Program Leg 147 at the Hess Deep. Paleomagnetic measurements indicate that samples acquired up to two small components of secondary remanent magnetization. Stable magnetic inclinations determined after alternating-field and thermal demagnetization reveal a mean stable magnetic direction $\left(38^{\circ}\right)$ that is significantly steeper than that predicted for this equatorial site $\left(<5^{\circ}\right)$. Thus, it is likely that remanent magnetization was acquired before tectonic uplift. The mean intensity of natural remanent magnetization for the recovered gabbros is $2.3 \mathrm{~A} / \mathrm{m}$, and the Koenigsberger ratio indicates that the in situ magnetization is dominated by remanent, rather than induced, magnetization. Measurements of hysteresis loop parameters indicate that the effective magnetic grain size of the gabbro samples falls within the pseudo-single domain region. Although recovered from a fast-spreading ridge, the paleomagnetic and rock magnetic properties of the gabbros from Hole $894 \mathrm{G}$ are very similar to those of gabbros recovered from slow-spreading ridges.
\end{abstract}

\section{INTRODUCTION}

Leg 147 penetrated several sections of tectonically exposed lower oceanic crust and shallow mantle at the Hess Deep in the equatorial Pacific. Hole $894 \mathrm{G}$ penetrated a total of 154 meters below seafloor $(\mathrm{mbsf})$ and recovered the first continuous section of gabbroic rock from a fast-spreading center. Thus, these gabbros represent an important opportunity to study the geological processes that form and, subsequently modify, lower oceanic crust in a fast-spreading environment. In this study, we present the results of paleomagnetic and rock magnetic measurements on gabbro samples taken from the $894 \mathrm{G}$ drill core. Following this, we characterize the magnetic structure of the Hole $894 \mathrm{G}$ gabbros, compare their magnetic properties to gabbros from slow-spreading centers, and evaluate the potential contribution of Hole $894 \mathrm{G}$ gabbros to marine magnetic anomalies.

\section{GEOLOGICAL SETTING}

The Hess Deep is the deepest part of a tectonic valley created by the westward propagation of the Cocos-Nazca spreading center into 1-Ma-old crust formed at the East Pacific Rise (Fig. 1; Lonsdale, 1988). The tectonic exposure of lower crustal and upper mantle rocks within the Hess Deep provided an opportunity to sample a representative section of lower crust/shallow mantle formed at a fast-spreading center in a location that is far from an oceanic fracture zone. Site 894 is located at a water depth of $3034 \mathrm{~m}$ on the summit of the intrarift ridge within the Hess Deep, and in a region where gabbros had previously been identified during Nautile and Alvin dive programs (Francheteau et al., 1990; Karson et al., 1992; Hekinian et al., 1993).

Hole $894 \mathrm{G}$ penetrated $154 \mathrm{~m}$ and recovered $49.09 \mathrm{~m}$ of drill core for an average recovery rate of $36 \%$. The majority of the recovered

'Mével, C., Gillis, K.M., Allan, J.F., and Meyer, P.S. (Eds.), 1996. Proc. ODP, Sci. Results, 147: College Station, TX (Ocean Drilling Program).

${ }^{2}$ School of Oceanography, University of Washington, Seattle, WA 98115 , U.S.A. pariso@washington.edu

${ }^{3}$ Lake Superior State University, Sault Ste. Marie, MI 49783, U.S.A.

${ }^{4}$ Ocean Drilling Program. Texas A\&M University Research Park, 1000 Discovery Drive, College Station, TX 77845-9547, U.S.A. drill core composed of gabbronorite $(77 \%)$, followed by olivine gabbronorite $(8 \%)$, basaltic dikes $(7 \%)$, small amounts of gabbro and olivine gabbro, and $<1 \%$ oxide gabbronorite (Fig. 2). Approximately $80 \%$ of the recovered gabbros are moderately altered to greenschist through amphibolite facies mineral assemblages. Small amounts of zeolite facies minerals are found throughout the core. The metamorphic assemblage and associated vein network indicate alteration occurred at temperatures ranging from $>500^{\circ} \mathrm{C}$ to ambient shallow crustal temperatures (Gillis, this volume; Lécuyer and Gruau, this volume).

\section{METHODS}

Measurements of natural remanent magnetization were made on minicores aboard the JOIDES Resolution using a 2-G cryogenic magnetometer, at the University of Minnesota using a Schonstedt spinner magnetometer, and at Texas A\&M University using a CTF cryogenic magnetometer. Stepwise alternating-field demagnetization (AFD) was performed using a Schonstedt Model GS-1 demagnetizer. Thermal demagnetization was performed using a Schonstedt Thermal Specimen Demagnetizer at steps of $100^{\circ}, 200^{\circ}, 300^{\circ}, 400^{\circ}, 450^{\circ}$, $500^{\circ}, 525^{\circ}, 550^{\circ}, 575^{\circ}$, and $600^{\circ} \mathrm{C}$. Individual components of remanent magnetization were identified using orthogonal vector diagrams (Zijderveld, 1967), and the directions of the individual components were calculated using a least-squares fitting routine. Mean inclination values were calculated using the method of McFadden and Reid (1982). Magnetic susceptibility was measured using a Kappa Bridge KLY-2 (Geofyzika Brno). Rock magnetic measurements were made at the Institute of Rock Magnetism at the University of Minnesota. Hysteresis loop parameters were measured on large (e.g., 5-30 g) chips using a Princeton Applied Research vibrating sample magnetometer (VSM) and on much smaller chips (e.g., 0.03-0.05 g) using a Princeton Applied Research alternating gradient force magnetometer. From two to five small chips were taken from each minicore to get a reasonable estimate of the average hysteresis loop parameters for each core. The agreement in values between the two magnetometers and different size samples was excellent. Curie temperature measurements were made using an oven attachment on the Princeton Applied Research VSM. 


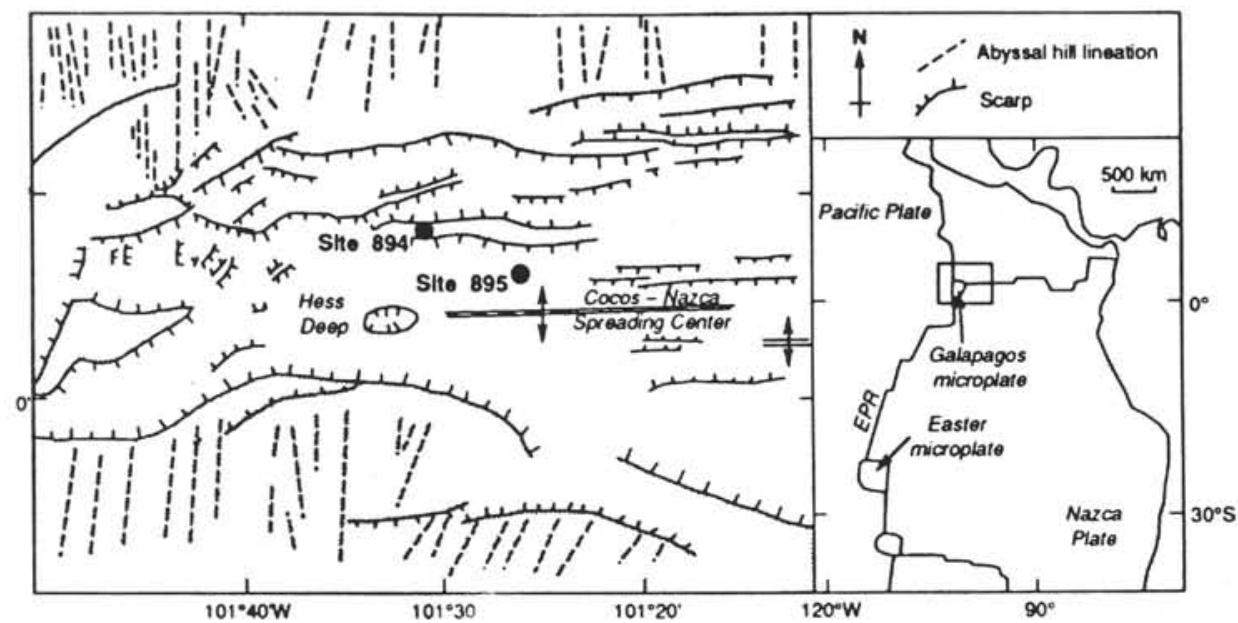

Figure 1. Location of Site 894 and Site 895 on the intrarift ridge within the Hess Deep.

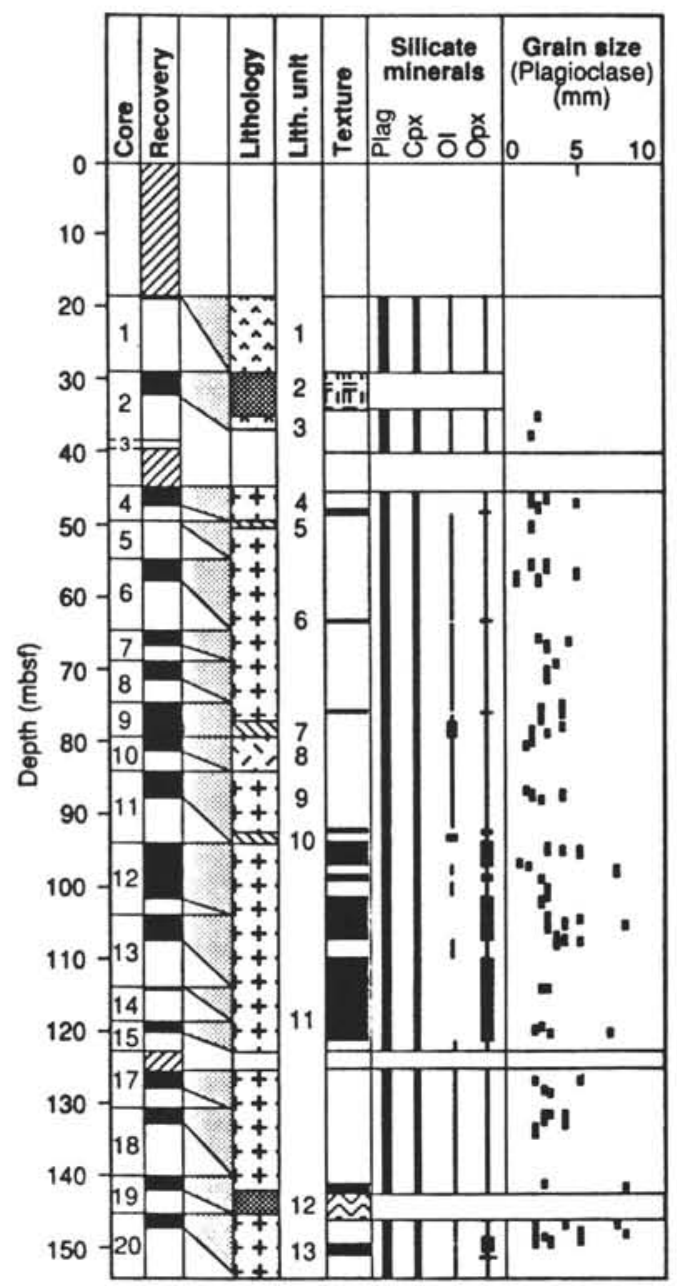

Recovery

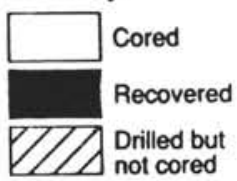

Lithology

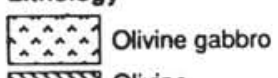

III Olivine

A.U. gabbronorite

Fi:1 Gabbro

Gabbronorite

Basaltic dikes

Texture



Silicate minerals

\begin{tabular}{|c|c|}
\hline Olivine & Orthopyroxe \\
\hline $\begin{array}{l}\text { May be } \\
\text { present } \\
\text { in small } \\
\text { amounts }\end{array}$ & $\begin{array}{l}\text { Present as } \\
\text { oikocrysts }\end{array}$ \\
\hline Present & $\begin{array}{l}\text { Present as } \\
\text { primocrysts }\end{array}$ \\
\hline
\end{tabular}

Figure 2. Summary of the igneous lithostratigraphy of Hole 894G. Plag = plagioclase, $\mathrm{Cpx}=$ clinopyroxene, $\mathrm{Ol}=$ olivine, and $\mathrm{Opx}=$ orthopyroxene. Grain size represents average plagioclase grain size (Shipboard Scientific Party, 1993). 
Table 1. Summary of paleomagnetic measurements for Hole 894G.

\begin{tabular}{|c|c|c|c|c|c|c|c|c|}
\hline $\begin{array}{l}\text { Core, section. } \\
\text { interval }(\mathrm{cm})\end{array}$ & $\begin{array}{l}\text { Depth } \\
\text { (mbsf) }\end{array}$ & $\begin{array}{l}\mathrm{NRM} \\
(\mathrm{A} / \mathrm{m})\end{array}$ & $\begin{array}{c}k \\
\text { (SI units) }\end{array}$ & Q & $\begin{array}{l}\text { MDF } \\
(\mathrm{mT})\end{array}$ & $\mathbf{1}_{n m m}$ & $1_{\text {stable }}$ & Rock type \\
\hline 147-894G- & & & & & & & & \\
\hline $2 \mathrm{R}-1,13-15$ & 19.03 & 0.75 & ND & ND & 26 & -40 & ND & Oxide gabbro \\
\hline $2 \mathrm{R}-1,20-22$ & 28.80 & 0.57 & ND & ND & 22 & 51 & 30 & Plag-olivine phyric basalt \\
\hline $2 \mathrm{R}-1,68-71$ & 29.28 & 0.66 & $8.75^{-3}$ & 2.87 & 23 & 36 & 27 & Diabase \\
\hline $2 \mathrm{R}-1,117-119$ & 29.77 & 1.50 & $9.50^{-3}$ & 6.03 & 17 & 16 & 21 & Plag-olivine phyric basalt \\
\hline $2 \mathrm{R}-2,57-59$ & 31.00 & 0.83 & $8.21^{-3}$ & 3.80 & ND & 32 & 30 & Plag-olivine phyric basalt \\
\hline $2 \mathrm{R}-2,110-113$ & 31.20 & 0.88 & $1.04^{-2}$ & 3.23 & 19 & 29 & 18 & Porphyritic diabase \\
\hline $2 \mathrm{R}-3,32-35$ & 31.87 & 0.07 & $2.62^{-3}$ & 1.08 & 36 & -2 & 3 & Deformed olivine gabbro \\
\hline $2 \mathrm{R}-3,118-121$ & 32.73 & 1.21 & $3.83^{-2}$ & 1.21 & 63 & 3 & ND & Gabbro \\
\hline $3 \mathrm{R}-1,6-8$ & 38.26 & 2.29 & $7.76^{-3}$ & 11.25 & 18 & -5 & 0 & Olivine Gabbro \\
\hline $4 \mathrm{R}-2,76-78$ & 47.10 & 0.25 & $9.37^{-4}$ & 10.18 & 17 & 68 & 68 & Gabbronorite \\
\hline $6 \mathrm{R}-1,26-28$ & 55.06 & 0.95 & $7.03^{-3}$ & 5.12 & 41 & 39 & 39 & Gabbronorite \\
\hline $6 \mathrm{R}-1,53-55$ & 55.33 & 1.01 & ND & ND & 38 & 35 & 38 & Gabbronorite \\
\hline $6 \mathrm{R}-1,77-79$ & 55.57 & 0.88 & $4.08^{-3}$ & 8.03 & ND & 21 & 24 & Gabbronorite \\
\hline $6 \mathrm{R}-1,89-91$ & 55.69 & 0.76 & $1.24^{-2}$ & 2.33 & 17 & 61 & 66 & Gabbronorite \\
\hline $6 \mathrm{R}-1,109-111$ & 55.89 & 0.64 & ND & ND & 44 & 58 & 63 & Gabbronorite \\
\hline $6 \mathrm{R}-2,0-2$ & 56.23 & 4.72 & $8.34^{-2}$ & 2.15 & 8 & 28 & 16 & Poikilitic gabbronorite \\
\hline $6 \mathrm{R}-2,43-45$ & 56.66 & 1.28 & ND & ND & 38 & 28 & 28 & Gabbronorite \\
\hline $6 \mathrm{R}-2,78-80$ & 57.01 & 1.76 & $1.84^{-2}$ & 3.64 & 13 & 42 & 30 & Olivine-bearing gabbronorite \\
\hline $6 \mathrm{R}-3,9-11$ & 57.82 & 1.80 & ND & ND & 8 & 49 & 42 & Gabbronorite \\
\hline $7 \mathrm{R}-1,99-101$ & 65.79 & 1.11 & $6.45^{-3}$ & 6.44 & ND & 41 & 27 & Gabbronorite \\
\hline $7 R-2,6-8$ & 66.26 & 1.72 & $1.16^{-2}$ & 5.65 & 20 & 41 & 32 & Olivine-bearing gabbronorite \\
\hline $7 \mathrm{R}-2,10-12$ & 66.30 & 1.83 & $1.09^{-2}$ & 6.29 & ND & 37 & 25 & Gabbronorite \\
\hline $7 \mathrm{R}-2,61-63$ & 66.80 & 1.07 & $4.91^{-3}$ & 8.12 & ND & 38 & 36 & Gabbronorite \\
\hline $8 \mathrm{R}-1,54-56$ & 69.04 & 0.69 & $1.32^{-2}$ & 2.01 & 13 & 29 & 26 & Altered gabbronorite \\
\hline $8 \mathrm{R}-2,3 \mathrm{l}-33$ & 70.31 & 2.14 & $1.12^{-2}$ & 7.29 & 34 & 38 & 34 & Gabbronorite \\
\hline $9 \mathrm{R}-1,120-122$ & 75.60 & 1.48 & $1.57^{-2}$ & 3.54 & ND & -55 & -31 & Gabbronorite \\
\hline $9 \mathrm{R}-2,17-19$ & 75.82 & 1.16 & ND & ND & 62 & 33 & 40 & Gabbronorite \\
\hline $9 R-2,66-68$ & 76.31 & 0.70 & ND & ND & 14 & 45 & 32 & Gabbronorite \\
\hline $9 \mathrm{R}-2,86-88$ & 76.51 & 1.67 & $1.62^{-2}$ & 3.93 & 44 & 50 & ND & Gabbronorite \\
\hline $9 \mathrm{R}-3,19-21$ & 77.10 & 1.92 & $1.98^{-2}$ & 3.63 & ND & 74 & 44 & Gabbronorite \\
\hline $9 \mathrm{R}-3,69-71$ & 77.60 & 1.34 & $3.91^{-2}$ & 1.30 & 4 & 49 & 12 & Altered gabbronorite \\
\hline 9R-3, 77-79 & 77.68 & 1.29 & $3.86^{-2}$ & 1.27 & 6 & 83 & 24 & Gabbronorite \\
\hline $9 \mathrm{R}-3,95-97$ & 77.86 & 3.08 & ND & ND & 7 & 57 & 35 & Olivine gabbronorite \\
\hline $9 \mathrm{R}-4,9-11$ & 78.42 & 0.20 & $5.26^{-3}$ & 1.43 & 16 & 53 & 49 & Olivine gabbronorite \\
\hline $9 R-4,45-47$ & 78.78 & 0.87 & $8.44^{-3}$ & 3.93 & 13 & 59 & 31 & Olivine gabbronorite \\
\hline $9 R-4,74-76$ & 79.07 & 1.30 & $1.29^{-2}$ & 3.84 & ND & 74 & 60 & Gabbronorite \\
\hline $9 R-4,95-97$ & 79.28 & 0.89 & $1.10^{-2}$ & 3.02 & ND & 75 & ND & Olivine gabbronorite \\
\hline $9 \mathrm{R}-4,130-132$ & 79.63 & 1.31 & $1.75^{-2}$ & 2.87 & 97 & 4 & ND & Gabbronorite \\
\hline $9 R-5,6-8$ & 79.83 & 0.58 & ND & ND & 19 & 75 & ND & Olivine gabbronorite \\
\hline $9 \mathrm{R}-5,12-14$ & 79.89 & 1.68 & ND & ND & 96 & 9 & ND & Olivine gabbronorite \\
\hline $10 \mathrm{R}-1,81-83$ & 79.91 & 2.19 & $1.52^{-2}$ & 5.48 & 63 & 35 & 36 & Gabbronorite \\
\hline $10 \mathrm{R}-1,91-93$ & 80.01 & 0.33 & $3.09^{-3}$ & 3.95 & ND & 46 & 39 & Gabbro \\
\hline $10 \mathrm{R}-1,124-126$ & 80.34 & 2.85 & ND & ND & 7 & 9 & 38 & Gabbro \\
\hline $10 \mathrm{R}-2,8-10$ & 80.56 & 1.89 & $1.32^{-2}$ & 5.35 & ND & 31 & 29 & Gabbro \\
\hline IIR-1,97-99 & 85.07 & 1.67 & ND & ND & 53 & 34 & 29 & Gabbronorite \\
\hline $11 R-2,34-6$ & 85.90 & 1.00 & $4.52^{-3}$ & 8.29 & ND & 31 & 31 & Gabbronorite \\
\hline $11 R-2,45-47$ & 86.01 & 1.14 & $7.55^{-3}$ & 5.73 & 71 & 43 & 35 & Altered gabbronorite \\
\hline IIR $-2,70-72$ & 86.26 & 1.65 & $1.62^{-2}$ & 3.88 & ND & ND & ND & Gabbro \\
\hline $12 \mathrm{R}-2,50-52$ & 95.76 & 4.71 & $3.01^{-2}$ & 5.97 & 73 & 54 & 44 & Olivine-bearing gabbronorite \\
\hline $12 \mathrm{R}-2,111-113$ & 96.37 & 4.83 & $3.20^{-2}$ & 5.65 & ND & 61 & 44 & Gabbronorite \\
\hline $12 \mathrm{R}-2,140-142$ & 96.66 & ND & $2.47^{-2}$ & ND & ND & ND & 54 & Gabbronorite \\
\hline $12 \mathrm{R}-3,4-6$ & 96.77 & 3.17 & $1.26^{-2}$ & 9.42 & ND & 41 & 36 & Gabbronorite \\
\hline $12 \mathrm{R}-3,30-32$ & 97.03 & 2.63 & ND & ND & 53 & 46 & 42 & Gabbronorite \\
\hline $12 \mathrm{R}-3,48-50$ & 97.21 & 2.99 & $1.09^{-2}$ & 10.27 & ND & 39 & 35 & Gabbronorite \\
\hline $12 \mathrm{R}-3,67-69$ & 97.40 & 2.51 & $8.23^{-3}$ & 11.60 & 51 & 39 & 35 & Poikilitic gabbronorite \\
\hline $12 R-3,90-92$ & 97.63 & 3.48 & ND & ND & 46 & 38 & 37 & Gabbronorite \\
\hline $12 \mathrm{R}-3,147-149$ & 98.20 & ND & $1.43^{-2}$ & ND & ND & ND & 36 & Olivine-bearing gabbronorite \\
\hline $12 \mathrm{R}-4,20-22$ & 98.43 & 3.74 & $1.67^{-2}$ & 8.39 & ND & 43 & 35 & Gabbronorite \\
\hline $\mid 2 R-4,51-53$ & 98.74 & ND & $1.90^{-2}$ & ND & ND & ND & 38 & Olivine-bearing gabbronorite \\
\hline $12 \mathrm{R}-4,139-141$ & 99.62 & 2.65 & $1.29^{-2}$ & 7.82 & 35 & 28 & 19 & Gabbro \\
\hline $12 R-5,64-66$ & 100.37 & 4.39 & $5.00^{-2}$ & 3.34 & ND & 60 & 41 & Olivine-bearing gabbronorite \\
\hline $12 R-5,79-81$ & 100.52 & 5.96 & $6.11^{-2}$ & 3.71 & 7 & 53 & 47 & Olivine-bearing gabbronorite \\
\hline $12 \mathrm{R}-5,100-102$ & 100.73 & 3.45 & $1.99^{-2}$ & 6.59 & ND & ND & ND & Gabbronorite \\
\hline $12 \mathrm{R}-5,116-118$ & 100.89 & 6.39 & $5.08^{-2}$ & 4.79 & ND & 72 & ND & Gabbronorite \\
\hline $12 \mathrm{R}-6,28-30$ & 101.45 & 2.04 & ND & ND & 57 & 46 & 39 & Gabbronorite \\
\hline $12 R-6,39-41$ & 101.56 & 5.19 & $5.14^{-2}$ & 3.78 & ND & 52 & 33 & Gabbronorite \\
\hline $13 \mathrm{R}-1,59-61$ & 103.99 & 3.67 & $3.05^{-2}$ & 4.51 & ND & 48 & 39 & Gabbronorite \\
\hline $13 \mathrm{R}-1,62-64$ & 104.02 & 4.67 & $1.48^{-2}$ & 11.82 & ND & 38 & 35 & Gabbronorite \\
\hline $13 R-2,58-60$ & 105.35 & 5.76 & ND & ND & 36 & 58 & 52 & Gabbronorite \\
\hline $13 \mathrm{R}-2,79-81$ & 105.69 & 2.62 & ND & ND & 35 & 52 & 45 & Gabbronorite \\
\hline $13 \mathrm{R}-2,120-122$ & 105.97 & 4.47 & $1.97^{\circ 2}$ & 8.65 & 44 & 48 & 43 & Gabbronorite \\
\hline $13 \mathrm{R}-2,142-144$ & 106.19 & 2.35 & $1.79^{-2}$ & 4.99 & ND & ND & ND & Gabbronorite \\
\hline $13 R-3,5-7$ & 106.28 & 7.20 & $4.42^{-2}$ & 6.20 & 20 & 59 & 44 & Gabbronorite \\
\hline $13 R-3,30-32$ & 106.53 & 3.67 & $1.85^{-2}$ & 7.43 & ND & 48 & 43 & Gabbronorite \\
\hline $13 \mathrm{R}-3,84-86$ & 107.07 & 3.58 & ND & ND & 36 & 40 & 37 & Gabbronorite \\
\hline $14 \mathrm{R}-1,70-72$ & 113.80 & ND & $1.31^{-2}$ & ND & ND & ND & 69 & Olivine-bearing gabbronorite \\
\hline $15 R-1,73-75$ & 119.53 & 3.82 & $1.97^{-2}$ & 7.37 & ND & 46 & 45 & Gabbronorite \\
\hline $16 \mathrm{R}-1,43-45$ & 123.23 & ND & $1.92^{-2}$ & ND & ND & ND & 2 & Gabbronorite \\
\hline $17 \mathrm{R}-1,60-62$ & 126.40 & 2.87 & $8.59^{-3}$ & 12.72 & 52 & 44 & 43 & Poikilitic gabbronorite \\
\hline $17 \mathrm{R}-1,90-92$ & 126.70 & 3.50 & $1.54^{-2}$ & 8.51 & ND & 39 & 41 & Gabbronorite \\
\hline $17 \mathrm{R}-1,123-125$ & 127.03 & 3.50 & $1.26^{-2}$ & 10.58 & 38 & 47 & 50 & Gabbronorite \\
\hline $17 \mathrm{R}-2,7-9$ & 127.31 & 2.67 & $1.45^{-2}$ & 7.00 & 38 & 44 & 51 & Gabbronorite \\
\hline $17 R-2,65-67$ & 127.89 & 1.86 & $3.35^{-3}$ & 21.13 & ND & 42 & ND & Poikilitic gabbronorite \\
\hline $17 R-2,114-116$ & 128.38 & 3.52 & $2.60^{-2}$ & 5.07 & ND & 34 & 42 & Gabbronorite \\
\hline $18 \mathrm{R}-1,69-71$ & 131.59 & 2.39 & $5.54^{-3}$ & 16.42 & 42 & 49 & 49 & Gabbronorite \\
\hline $18 \mathrm{R}-2.7-9$ & 132.39 & 2.03 & $4.45^{-3}$ & 17,40 & 39 & 47 & 44 & Poikilitic gabbronorite \\
\hline $18 \mathrm{R}-2,94-96$ & 133.26 & 0.27 & $7.48^{-3}$ & 1.40 & 22 & 55 & 65 & Altered gabbronorite \\
\hline $18 \mathrm{R}-2,110-112$ & 133.42 & 2.45 & ND & ND & 39 & 36 & 37 & Gabbronorite \\
\hline
\end{tabular}


Table 1 (continued).

\begin{tabular}{|c|c|c|c|c|c|c|c|c|c|}
\hline $\begin{array}{l}\text { Core, section, } \\
\text { interval }(\mathrm{cm})\end{array}$ & $\begin{array}{l}\text { Depth } \\
\text { (mbsf) }\end{array}$ & $\begin{array}{l}\mathrm{NRM} \\
(\mathrm{A} / \mathrm{m})\end{array}$ & $\begin{array}{c}\mathrm{k} \\
\text { (SI units) }\end{array}$ & Q & $\begin{array}{l}\mathrm{MDF} \\
(\mathrm{mT})\end{array}$ & $1_{n m m}$ & $\mathrm{I}_{\text {tatable }}$ & Rock type & Source lab \\
\hline $19 \mathrm{R}-1,37-39$ & 140.87 & 0.60 & ND & ND & 48 & -7 & ND & Gabbronorite & U.W. \\
\hline $19 R-1,72-74$ & 141.22 & 0.03 & $6.07^{-4}$ & 1.92 & 17 & -11 & ND & Phyric basalt & J.R. \\
\hline $20 \mathrm{R}-1,60-62$ & 146.20 & ND & $1.36^{2}$ & ND & ND & ND & 44 & Gabbronorite & \\
\hline $20 \mathrm{R}-1,92-94$ & 146.52 & 2.67 & ND & ND & 59 & 39 & 32 & Gabbronorite & U.W. \\
\hline $20 \mathrm{R}-1,117-119$ & 146.77 & 2.25 & $2.08^{-2}$ & 4.12 & ND & ND & ND & Poikilitic gabbronorite & U.M. \\
\hline $20 \mathrm{R}-3.56-58$ & 147.98 & ND & $2.37^{-2}$ & ND & ND & ND & ND & Gabbronorite & \\
\hline $20 \mathrm{R}-3,124-126$ & 148.66 & 2.91 & $6.59^{-3}$ & 16.52 & ND & -31 & -31 & Gabbronorite & TAMU \\
\hline 20R-3, 107-109 & $\begin{array}{l}140.00 \\
149.70\end{array}$ & 0.50 & $2.43^{-2}$ & 0.78 & ND & ND & ND & Poikilitic gabbronorite & U.M. \\
\hline
\end{tabular}

Notes: $\mathrm{ND}=$ particular parameter not determined. The labs in which individual samples were analyzed are as follows: J.R. = JOIDES Resolution, U.W. = University of Washington, U.M. = University of Minnesota, and TAMU = Texas A\&M University.

Table 2. Summary of rock magnetic measurements for Hole 894G.

\begin{tabular}{|c|c|c|c|c|c|c|}
\hline $\begin{array}{l}\text { Core, section, } \\
\text { interval }(\mathrm{cm})\end{array}$ & Subsample & $\begin{array}{l}\text { Depth } \\
\text { (mbsf) }\end{array}$ & $\frac{J_{\mathrm{s}}}{\left(\mathrm{Am}^{2} \mathrm{~kg}^{-1}\right)}$ & $J_{r s} / J_{s}$ & $\begin{array}{c}H_{c} \\
(\mathrm{mT})\end{array}$ & $\mathrm{H}_{r c} / \mathrm{H}_{c}$ \\
\hline \multicolumn{7}{|l|}{ 147-894G- } \\
\hline $2 \mathrm{R}-1,20-22$ & a & 28.80 & 0.598 & 0.31 & 32.5 & 1.6 \\
\hline 2R-1, 20-22 & b & 28.80 & 0.867 & 0.33 & 33.2 & 1.5 \\
\hline $2 \mathrm{R}-1,20-22$ & c & 28.80 & 0.592 & 0.31 & 32.4 & 1.6 \\
\hline $2 \mathrm{R}-1,20-22$ & d & 28.80 & 0.700 & 0.31 & 32.0 & 1.6 \\
\hline $2 \mathrm{R}-2,57-59$ & & 30.67 & 0.332 & 0.34 & 27.5 & 1.6 \\
\hline $6 \mathrm{R}-1,53-55$ & a & 55.33 & 0.200 & 0.14 & 13.7 & 2.7 \\
\hline $6 \mathrm{R}-1,53-55$ & b & 55.33 & 0.168 & 0.13 & 12.7 & 2.9 \\
\hline $6 \mathrm{R}-1,53-55$ & c & 55.33 & 1.520 & 0.09 & 3.4 & 5.3 \\
\hline $6 \mathrm{R}-1,53-55$ & d & 55.33 & 0.354 & 0.10 & 8.6 & 3.4 \\
\hline $6 \mathrm{R}-1,53-55$ & $\mathrm{e}$ & 55.33 & 0.172 & 0.11 & 9.9 & 3.9 \\
\hline $6 \mathrm{R}-1,77-79$ & & 55.57 & 0.195 & 0.14 & 11.2 & 3.2 \\
\hline $6 \mathrm{R}-1,109-111$ & a & 55.89 & 0.104 & 0.16 & 14.6 & 2.9 \\
\hline $6 \mathrm{R}-1,109-111$ & b & 55.89 & 0.030 & 0.26 & 30.1 & 2.2 \\
\hline $6 \mathrm{R}-1,109-111$ & c & 55.89 & 0.089 & 0.16 & 14.5 & 3.3 \\
\hline $6 \mathrm{R}-1,109-111$ & d & 55.89 & 0.316 & 0.08 & 9.7 & 3.6 \\
\hline $6 \mathrm{R}-2,43-45$ & a & 56.66 & 0.204 & 0.12 & 11.1 & 3.5 \\
\hline $6 \mathrm{R}-2,43-45$ & b & 56.66 & 0.384 & 0.07 & 7.2 & 5.0 \\
\hline $6 \mathrm{R}-2,43-45$ & c & 56.66 & 0.132 & 0.14 & 14.6 & 3.0 \\
\hline $7 R-2,10-12$ & & 66.30 & 0.398 & 0.10 & 5.8 & 3.5 \\
\hline $9 \mathrm{R}-2,17-19$ & a & 75.82 & 0.188 & 0.10 & 11.6 & 3.5 \\
\hline $9 \mathrm{R}-2,17-19$ & b & 75.82 & 0.115 & 0.17 & 16.9 & 2.8 \\
\hline $9 \mathrm{R}-2,17-19$ & c & 75.82 & 0.187 & 0.11 & 10.3 & 4.4 \\
\hline $9 \mathrm{R}-2,17-19$ & d & 75.82 & 0.162 & 0.13 & 11.9 & 3.2 \\
\hline $9 R-2,66-68$ & a & 76.31 & 0.043 & 0.15 & 15.7 & 2.9 \\
\hline 9R-2, 66-68 & b & 76.31 & 0.058 & 0.10 & 10.8 & 4.3 \\
\hline 9R-2, 66-68 & $\mathrm{c}$ & 76.31 & 0.624 & 0.06 & 4.7 & 4.2 \\
\hline 9R-3, 77-79 & & 77.68 & 2.220 & 0.02 & 2.7 & 4.9 \\
\hline $9 R-3,95-97$ & a & 77.86 & 0.350 & 0.19 & 14.4 & 2.2 \\
\hline $9 R-3,95-97$ & b & 77.86 & 0.682 & 0.16 & 10.9 & 2.2 \\
\hline 9R-3, 95-97 & c & 77.86 & 0.708 & 0.12 & 9.2 & 3.3 \\
\hline $9 \mathrm{R}-4,9-11$ & & 78.42 & 0.138 & 0.15 & 10.2 & 2.4 \\
\hline $9 R-4,95-97$ & & 79.28 & 0.540 & 0.15 & 8.1 & 2.6 \\
\hline $9 R-5,6-8$ & a & 79.83 & 0.022 & 0.17 & 13.8 & 3.5 \\
\hline $9 R-5,6-8$ & b & 79.83 & 1.050 & 0.19 & 10.2 & 1.9 \\
\hline $9 R-5,6-8$ & $\mathrm{c}$ & 79.83 & 0.017 & 0.18 & 9.7 & 2.1 \\
\hline $9 R-5,6-8$ & d & 79.83 & 0.012 & 0.11 & 11.0 & 2.8 \\
\hline $9 R-5,12-14$ & a & 79.89 & 0.338 & 0.21 & 17.8 & 2.1 \\
\hline $9 \mathrm{R}-5,12-14$ & b & 79.89 & 0.364 & 0.10 & 7.1 & 5.2 \\
\hline $9 R-5,12-14$ & c & 79.89 & 0.337 & 0.17 & 13.6 & 2.6 \\
\hline $9 \mathrm{R}-5,12-14$ & d & 79.89 & 4.710 & 0.07 & 6.7 & 2.8 \\
\hline $10 \mathrm{R}-1,124-126$ & a & 80.34 & 0.327 & 0.07 & 6.9 & 4.1 \\
\hline $10 \mathrm{R}-1,124-126$ & b & 80.34 & 0.341 & 0.08 & 6.2 & 3.9 \\
\hline $10 \mathrm{R}-1,124-126$ & c & 80.34 & 0.209 & 0.09 & 8.6 & 4.1 \\
\hline $10 \mathrm{R}-1,124-126$ & d & 80.34 & 0.391 & 0.07 & 6.4 & 3.7 \\
\hline $10 \mathrm{R}-2,8-10$ & & 80.56 & 0.382 & 0.07 & 6.0 & 4.4 \\
\hline $11 \mathrm{R}-2,34-36$ & & 85.90 & 0.140 & 0.09 & 6.3 & 4.8 \\
\hline
\end{tabular}

\begin{tabular}{|c|c|c|c|c|c|c|}
\hline $\begin{array}{l}\text { Core, section, } \\
\text { interval }(\mathrm{cm})\end{array}$ & Subsample & $\begin{array}{l}\text { Depth } \\
\text { (mbsf) }\end{array}$ & $\begin{array}{c}J_{\mathrm{s}} \\
\left(\mathrm{Am}^{2} \mathrm{~kg}^{-1}\right)\end{array}$ & $J_{r s} / J_{s}$ & $\begin{array}{c}H_{c} \\
(\mathrm{mT})\end{array}$ & $\mathrm{H}_{r e} / \mathrm{H}_{c}$ \\
\hline $1 \mathrm{IR}-2,70-72$ & & 86.26 & 1.330 & 0.05 & 4.5 & 3.3 \\
\hline $12 \mathrm{R}-2,140-142$ & & 96.66 & 0.487 & 0.09 & 6.3 & 3.6 \\
\hline $12 \mathrm{R}-3,4-6$ & & 96.77 & 0.811 & 0.09 & 4.4 & 3.4 \\
\hline $12 \mathrm{R}-3,30-32$ & a & 97.03 & 0.207 & 0.15 & 12.6 & 2.5 \\
\hline $12 \mathrm{R}-3,30-32$ & b & 97.03 & 0.331 & 0.13 & 11.7 & 3.1 \\
\hline $12 \mathrm{R}-3,30-32$ & c & 97.03 & 0.283 & 0.12 & 10.9 & 3.3 \\
\hline $12 \mathrm{R}-3,30-32$ & d & 97.03 & 0.283 & 0.13 & 12.3 & 3.3 \\
\hline $12 \mathrm{R}-3,48-50$ & & 97.21 & 0.157 & 0.12 & 9.4 & 0.4 \\
\hline $12 \mathrm{R}-3,67-69$ & & 97.40 & 0.286 & 0.10 & 7.2 & 3.8 \\
\hline $12 \mathrm{R}-3,90-92$ & a & 97.63 & 0.142 & 0.16 & 17.8 & 2.8 \\
\hline $12 \mathrm{R}-3,90-92$ & b & 97.63 & 0.298 & 0.10 & 9.8 & 4.3 \\
\hline $12 \mathrm{R}-3,90-92$ & $\mathrm{c}$ & 97.63 & 0.272 & 0.12 & 12.2 & 3.2 \\
\hline $12 \mathrm{R}-4,20-22$ & & 98.43 & 0.573 & 0.12 & 5.6 & 0.5 \\
\hline$|2 R-4,139-14|$ & & 99.62 & 0.449 & 0.09 & 6.4 & 3.1 \\
\hline $12 \mathrm{R}-5,100-101$ & & 100.73 & 0.503 & 0.08 & 5.1 & 4.0 \\
\hline $12 \mathrm{R}-6,28-30$ & a & 101.45 & 0.179 & 0.20 & 17.6 & 2.0 \\
\hline $12 \mathrm{R}-6,28-30$ & b & 101.45 & 0.196 & 0.17 & 13.4 & 2.2 \\
\hline $12 \mathrm{R}-6,28-30$ & c & 101.45 & 0.151 & 0.14 & 16.0 & 3.0 \\
\hline $12 \mathrm{R}-6,28-30$ & d & 101.45 & 0.226 & 0.13 & 10.7 & 2.8 \\
\hline $12 \mathrm{R}-6,39-41$ & & 101.56 & 1.650 & 0.06 & 4.8 & 3.6 \\
\hline $13 R-2,58-60$ & a & 105.35 & 1.580 & 0.02 & 2.6 & 8.0 \\
\hline $13 \mathrm{R}-2,58-60$ & b & 105.35 & 0.437 & 0.18 & 10.2 & 0.9 \\
\hline $13 R-2,58-60$ & c & 105.35 & 5.750 & 0.03 & 1.9 & 5.2 \\
\hline $13 R-2,58-60$ & d & 105.35 & 0.151 & 0.13 & 11.9 & 4.4 \\
\hline $13 \mathrm{R}-2,58-60$ & e & 105.35 & 0.374 & 0.09 & 9.4 & 3.5 \\
\hline $13 \mathrm{R}-2,79-8 \mathrm{I}$ & a & 105.56 & 0.256 & 0.09 & 4.2 & 6.0 \\
\hline $13 \mathrm{R}-2,79-81$ & b & 105.56 & 0.149 & 0.17 & 14.4 & 2.9 \\
\hline $13 \mathrm{R}-2,79-81$ & c & 105.56 & 1.140 & 0.19 & 6.0 & 2.4 \\
\hline $13 \mathrm{R}-2,79-81$ & d & 105.56 & 0.211 & 0.08 & 7.0 & 6.1 \\
\hline $13 \mathrm{R}-2,120-122$ & & 105.97 & 0.456 & 0.09 & 5.4 & 3.2 \\
\hline $13 R-2,142-144$ & & 106.19 & 0.697 & 0.08 & 6.3 & 3.2 \\
\hline $13 R-3,30-32$ & & 106.53 & 0.363 & 0.11 & 7.9 & 2.9 \\
\hline $17 \mathrm{R}-2,114-116$ & & 128.38 & 0.180 & 0.09 & 5.7 & 4.8 \\
\hline 18R-2, 7-9 & & 132.39 & 0.128 & 0.12 & 9.6 & 3.9 \\
\hline $18 \mathrm{R}-2,110-112$ & a & 133.42 & 0.111 & 0.11 & 12.5 & 3.6 \\
\hline $18 \mathrm{R}-2,110-112$ & b & 133.42 & 0.135 & 0.08 & 9.9 & 4.0 \\
\hline $19 \mathrm{R}-1,37-39$ & a & 140.87 & 0.125 & 0.15 & 15.4 & 3.3 \\
\hline $19 \mathrm{R}-1,37-39$ & b & 140.87 & 0.113 & 0.12 & 12.6 & 3.9 \\
\hline $19 \mathrm{R}-1,37-39$ & c & 140.87 & 0.178 & 0.12 & 12.9 & 3.5 \\
\hline $20 \mathrm{R}-1,92-94$ & a & 146.52 & 0.166 & 0.17 & 61.6 & 0.8 \\
\hline $20 \mathrm{R}-1.92-94$ & b & 146.52 & 1.520 & 0.09 & 6.0 & 3.7 \\
\hline $20 \mathrm{R}-1,92-94$ & c & 146.52 & 0.493 & 0.16 & 12.9 & 2.3 \\
\hline $20 \mathrm{R}-1,92-94$ & d & 146.52 & 0.297 & 0.08 & 9.7 & 4.6 \\
\hline $21 \mathrm{R}-1,92-94$ & d & 146.52 & 0.341 & 0.11 & 8.1 & 2.9 \\
\hline $20 \mathrm{R}-1,117-119$ & & 146.77 & 0.296 & 0.02 & 5.4 & 4.5 \\
\hline $20 \mathrm{R}-3,124-126$ & & 148.66 & 0.237 & 0.13 & 10.7 & 3.6 \\
\hline $20 \mathrm{R}-3,107-109$ & & 149.7 & 0.483 & 0.13 & 7.7 & 2.4 \\
\hline
\end{tabular}

Note: $\mathrm{ND}=$ particular parameter not determined.

\section{RESULTS}

Paleomagnetic and rock magnetic data for Leg 147 samples are displayed in Tables 1 and 2, respectively. Mean values of paleomagnetic and rock magnetic parameters for Leg 147 gabbros are displayed in Table 3. For comparison, Table 3 also summarizes mean values of paleomagnetic and rock magnetic parameters for (1) gabbros drilled and dredged from the Mid-Atlantic Ridge (compiled from Fox and Opdyke, 1973; Kent et al., 1978; and Dunlop and Prevot, 1982) and (2) olivine gabbros and gabbronorites recovered from ODP Hole 735B at the Southwest Indian Ridge (Pariso and Johnson, 1993a, 1993b).

\section{Remanent Magnetic Properties}

Values of the intensity of natural remanent magnetization $\left(J_{0}\right)$ are plotted vs. depth for Hole $894 \mathrm{G}$ in Figure 3 . The $J_{0}$ values range over 2 orders of magnitude with a mean of $2.3 \mathrm{~A} / \mathrm{m}$ for gabbro samples. In comparison, this value is similar to olivine gabbros and gabbronorites from ODP Hole 735B (Pariso and Johnson, 1993a) and somewhat higher than the average $J_{0}$ value $(1.2 \mathrm{~A} / \mathrm{m})$ for gabbros from the MidAtlantic Ridge (Dunlop and Prevot, 1982; Fox and Opdyke, 1973; Kent et al., 1978). The highest $J_{0}$ values are observed at about 100 mbsf in samples from Cores 147-894G-12R and 13R. These cores are from lithologic Unit 11 (see Fig. 2), a gabbronorite unit with primary 
orthopyroxene phenocrysts and abundant primary magnetite. The susceptibility $(k)$ values for Hole $894 \mathrm{G}$ gabbros are plotted vs. depth in Figure 3 . The mean $k$ value is 0.018 SI. This value is very close to the $k$ value observed in gabbros from slow-spreading ridges, the MidAtlantic Ridge, and Hole 735B on the Southwest Indian Ridge.

The Koenigsberger $(Q)$ ratio compares the magnitude of the remanent magnetization to the instantaneous magnetization induced in the sample by the Earth's field. $Q$ values were calculated for Hole $894 \mathrm{G}$ samples using the equation $Q=J_{0}(k \times H)$, where $H$ is the value of the geomagnetic field at Site $894(0.033 \mathrm{mT})$. Figure 3 shows that nearly all of the samples have values much greater than 1 and are thus dominated by remanent, rather than induced, magnetization. Because it is the remanent magnetization that records reversals of the Earth's magnetic field, this becomes a particularly important fact to establish when assessing the ability of this crustal section to contribute to marine magnetic anomalies.

The NRM of the Hole $894 \mathrm{G}$ gabbros was generally very resistant to alternating-field demagnetization. Examples of typical demagnetization curves are shown in Figure 4. In several cases, as much as $40 \%$ of the initial remanent magnetization remained after demagnetization at $\mathrm{AF}$ levels of $100 \mathrm{mT}$. A simple quantitative way to compare the demagnetization behavior of individual samples is to calculate the demagnetizing field required to remove half of the NRM. This parameter is known as the median demagnetizing field (MDF). MDF values for the Hole 894G gabbro samples are plotted in Figure 3. The mean MDF value is $31.0 \mathrm{mT}$, which again demonstrates that most of the gabbros are resistant to $\mathrm{AF}$ demagnetization. This high stability against AF demagnetization indicates that the remanent magnetization of Hole $894 \mathrm{G}$ gabbros is likely to be stable over geologically long periods of time. The MDF values are comparable to those observed on gabbros from the Mid-Atlantic Ridge (Fox and Opdyke, 1973; Kent et al., 1978; Dunlop and Prevot, 1982). Unfortunately, we cannot compare the demagnetization results from Hole $894 \mathrm{G}$ gabbros with those from Hole $735 \mathrm{~B}$ because the initial remanence of the Hole $735 \mathrm{~B}$ gabbros was modified by a strong, but soft drilling-induced remanent magnetization (Kikawa and Pariso, 1991; Pariso and Johnson, 1993a).

\section{Direction of Remanent Magnetization}

Values of the inclination of NRM $\left(I_{n r m}\right)$ and stable remanent magnetization $\left(I_{\text {stabie }}\right)$ are plotted vs. depth in Figure 5. The mean $I_{n m m}$ and $I_{\text {stable }}$ values for the Hole $894 \mathrm{G}$ gabbro samples are $45^{\circ}$ and $38^{\circ}$, respectively, indicating that the in situ direction of remanent magnetization is very similar to the stable remanent magnetization. One or two small components of secondary magnetization were commonly observed (Fig. 6). Because these components were small (often removed by $2.5 \mathrm{mT}$ ) and there is no azimuthal control of the cores, it is difficult to assess the origin of the secondary components. However, there was no apparent consistency of the inclination of the secondary components, suggesting that they were not caused by a systematic geological process. There is a slight steepening (about $10^{\circ}$ ) of $\mathrm{I}_{\text {stable }}$ values with depth. This may be caused by an increasing tilt of the borehole with depth. Unfortunately, bad hole conditions restricted logging to a relatively short section between 36 and 72 mbsf. Thus, although we have an estimate of the borehole tilt in this section, we are unable to determine if the tilt of the borehole increases below a depth of 72 mbsf.

Based on a geocentric axial dipole field, the magnetic inclination expected for Site 894 is $<5^{\circ}$. Thus, the observed inclination of stable remanent magnetization for Hole $894 \mathrm{G}$ samples is significantly steeper than the predicted magnetic inclination. For equatorial latitudes, up to $13^{\circ}$ of angular dispersion in magnetic direction are predicted based on variations in the Earth's field during the past $5 \mathrm{Ma}$ (McFadden and McElhinny, 1984). Continuous measurements of the tilt of the borehole between 36 and 72 mbsf indicated that Hole 895G
Table 3. Magnetic properties of oceanic gabbros.

\begin{tabular}{|c|c|c|c|c|}
\hline & & Mean & SD & $\begin{array}{c}\text { Samples } \\
(N)\end{array}$ \\
\hline \multicolumn{5}{|l|}{$J_{0}(\mathrm{~A} / \mathrm{m})$} \\
\hline & Hole 894G & 2.3 & 1.6 & 84 \\
\hline & Mid-Atlantic Ridge & 1.2 & 3.4 & 94 \\
\hline & Hole 735B & 2.2 & 2.5 & 113 \\
\hline \multicolumn{5}{|l|}{$k$ (SI units) } \\
\hline & Hole 894G & 0.018 & 0.015 & 70 \\
\hline & Mid-Atlantic Ridge & 0.018 & 0.037 & 92 \\
\hline & Hole 735B & 0.011 & 0.029 & 120 \\
\hline \multicolumn{5}{|c|}{ Hel $804 \mathrm{C}$} \\
\hline & Hole 894G & 5.9 & 3.6 & 61 \\
\hline & Mid-Atlantic Ridge & 6.0 & 11.0 & $\begin{array}{r}89 \\
117\end{array}$ \\
\hline & Hole 735B & 9.9 & 8.5 & 117 \\
\hline \multicolumn{5}{|l|}{$\mathrm{MDF}(\mathrm{mT})$} \\
\hline & Hole $894 \mathrm{G}$ & 31.0 & 19.0 & $\begin{array}{l}53 \\
65\end{array}$ \\
\hline & $\begin{array}{l}\text { Mid-Atlantic Ridge } \\
\text { Hole 735B }\end{array}$ & $\begin{array}{r}28.0 \\
\text { ND }\end{array}$ & $\begin{array}{r}17.0 \\
\text { ND }\end{array}$ & $\begin{array}{r}65 \\
\text { ND }\end{array}$ \\
\hline \multicolumn{5}{|c|}{$J_{s}\left(\mathrm{Am}^{2} \mathrm{~kg}^{-1}\right)$} \\
\hline & Hole 894G & 0.50 & 0.83 & 88 \\
\hline & Mid-Atlantic Ridge & 0.49 & 0.76 & 20 \\
\hline & Hole 735B & 0.17 & 0.16 & 54 \\
\hline \multicolumn{5}{|l|}{$J_{r} / J_{s}$} \\
\hline & $\begin{array}{l}\text { Hole } 894 \mathrm{G} \\
\text { Mid-Atlantic Ridoe }\end{array}$ & 0.12 & $\begin{array}{l}0.05 \\
0.13\end{array}$ & $\begin{array}{l}88 \\
18\end{array}$ \\
\hline & Hole 735B & $\begin{array}{l}0.22 \\
0.16\end{array}$ & $\begin{array}{l}0.13 \\
0.07\end{array}$ & $\begin{array}{l}18 \\
54\end{array}$ \\
\hline \multicolumn{5}{|l|}{$H_{c}(\mathrm{mT})$} \\
\hline & Hole $894 \mathrm{G}$ & 10.5 & 7.0 & 88 \\
\hline & Mid-Atlantic Ridge & 16.0 & 13.0 & 18 \\
\hline & Hole 735B & 11.7 & 4.2 & 54 \\
\hline
\end{tabular}

Notes: Data for Hole $894 \mathrm{G}$ are from the present study. Mid-Atlantic Ridge gabbro data were compiled from Dunlop and Prevot (1982) and Kent et al. (1978). Hole 735B data for olivine gabbros and gabbronorites were compiled from Pariso and Johnson (1993a), $\mathrm{SD}=$ standard deviation, $N=$ number of samples, and $\mathrm{ND}=$ not determined.

is inclined $\sim 4^{\circ}$. Thus, it is unlikely that either normal variations of the Earth's field or tilt of the borehole are responsible for the steep $\mathrm{I}_{\text {stablc }}$ values. These observations demonstrate that it is unlikely that this crustal section acquired its remanent magnetization in its in situ position. Rather, we suggest that it experienced significant tectonic rotation (up to $45^{\circ}$ ) about a horizontal axis after the acquisition of a near-horizontal remanent magnetization. This model is consistent with the observation that these rocks have undergone considerable tectonic uplift (e.g., Lonsdale, 1988; Francheteau et al., 1990). Because ODP drill cores are not azimuthally oriented, we are unable to determine if significant horizontal rotation about a vertical axis took place. The possible mechanisms of rotation and uplift of this crustal section, and their geographic context with respect to the East Pacific Rise were analyzed, using paleomagnetic data, deformation structures in the recovered drill core, and borehole televiewer data, and discussed by MacLeod, Célérier, et al. (this volume).

\section{Rock Magnetic Properties}

Hysteresis loop parameters were measured on 42 of the Leg 147 gabbro samples to examine the intrinsic magnetic properties of these rocks and the variation in effective magnetic grain size within the drilled gabbros. Saturation magnetization $\left(J_{s}\right)$ measures the total quantity of magnetic material in the sample (minus the paramagnetic component) and is plotted vs. depth in Figure $7 . J_{s}$ is typically dependent on both the concentration and composition of the magnetic material within a sample. As will be discussed later, the dominant magnetic material in the Hole $894 \mathrm{G}$ samples is low-titanium magnetite. In this case, the $J_{s}$ values can be directly related to the total quantity of magnetite within the individual samples. Figure 7 shows $J_{s}$ values of Hole $894 \mathrm{G}$ samples plotted vs. depth in the drilled section. The mean value of $J_{s}$ for the gabbro samples is $0.50 \mathrm{~A} \mathrm{~m}^{2} / \mathrm{kg}$. This value is very close to the mean $J_{s}$ value observed for gabbros from the Mid- 


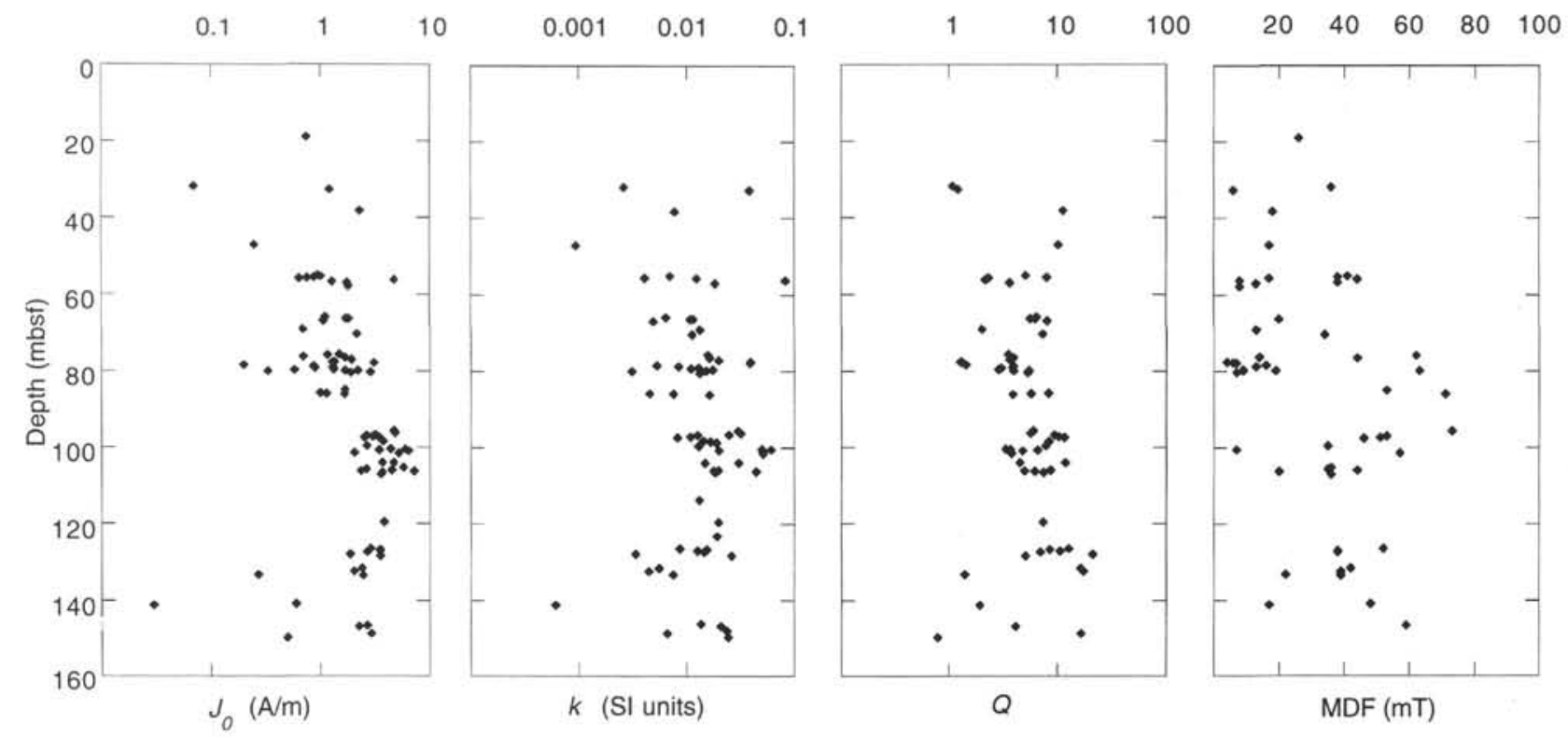

Figure 3. Intensity of NRM $\left(J_{0}\right)$, magnetic susceptibility $(k), Q$ ratio, and mean demagnetization field (MDF) vs. depth for Hole $894 \mathrm{G}$ samples. Note that $J_{0}, k$, and $Q$ are plotted on logarithmic scales.


Figure 4. Normalized magnetization vs. AF demagnetization field shows typical decay of NRM for two Hole $894 \mathrm{G}$ gabbro samples. A. Sample $147-$ 894G-13R-2, 58-60 cm. B. Sample 147-894G-20R-1, 94-96 cm.

Atlantic Ridge (Dunlop and Prevot, 1982; Fox and Opdyke, 1973; Kent et al., 1978). In comparison, the mean $J_{s}$ value for olivine gabbros and gabbronorites from Hole $735 \mathrm{~B}$ is somewhat lower $(0.17$ $\left.\mathrm{Am}^{2} \mathrm{~kg}^{-1}\right)$. Figure 7 also shows that the highest $J_{s}$ values are observed at about $100 \mathrm{mbsf}$. As previously discussed, $J_{\theta}$ values are also high at

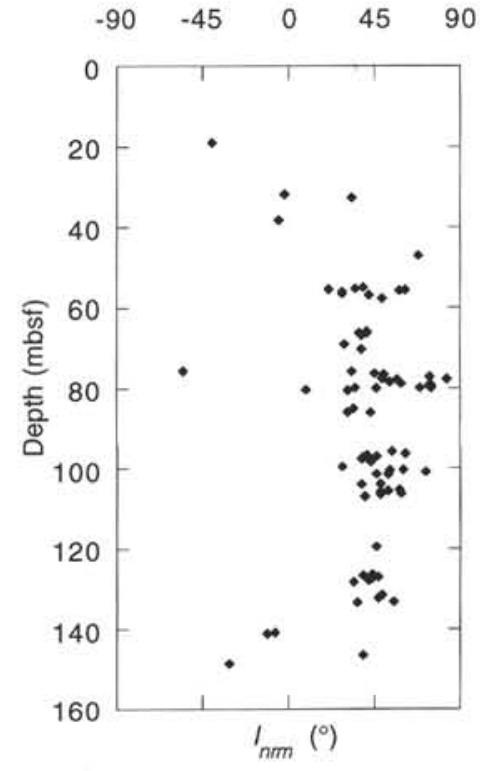

$\begin{array}{llllll}90 & -90 & -45 & 0 & 45 & 90\end{array}$

Figure 5. Inclination of natural remanent magnetization $\left(I_{n r m}\right)$ and stable remanent magnetization $\left(I_{\text {stable }}\right)$ vs. depth for Hole $894 \mathrm{G}$ gabbro samples.

this depth and correspond to a gabbronorite unit with primary orthopyroxene phenocrysts and abundant primary magnetite.

The ratio of saturation magnetization to saturation remanence $\left(J_{r s} J\right.$ $J_{s}$ ) provides a quantitative measure of the proportion of magnetic minerals that are capable of carrying a stable remanent magnetization. This ratio is commonly used to estimate the effective magnetic grain size (e.g., Day et al., 1977), with $J_{r s} / J_{s}$ values less than 0.1 indicating that the carrier of remanent magnetization is multidomain and with $J_{r s} / J_{s}$ values greater than 0.5 indicating that the carrier of remanent magnetization is dominated by single-domain grains (Dunlop, 1973). Figure 7 shows values of $J_{s} / J_{s}$ for the Hole $894 \mathrm{G}$ samples plotted vs. depth. The mean $J_{r s} / J_{s}$ value $(0.12)$ indicates that the majority of the Hole $894 \mathrm{G}$ gabbro samples exhibit pseudo-single domain behavior (Fig. 8). Thus, although the average magnetic grain 
A

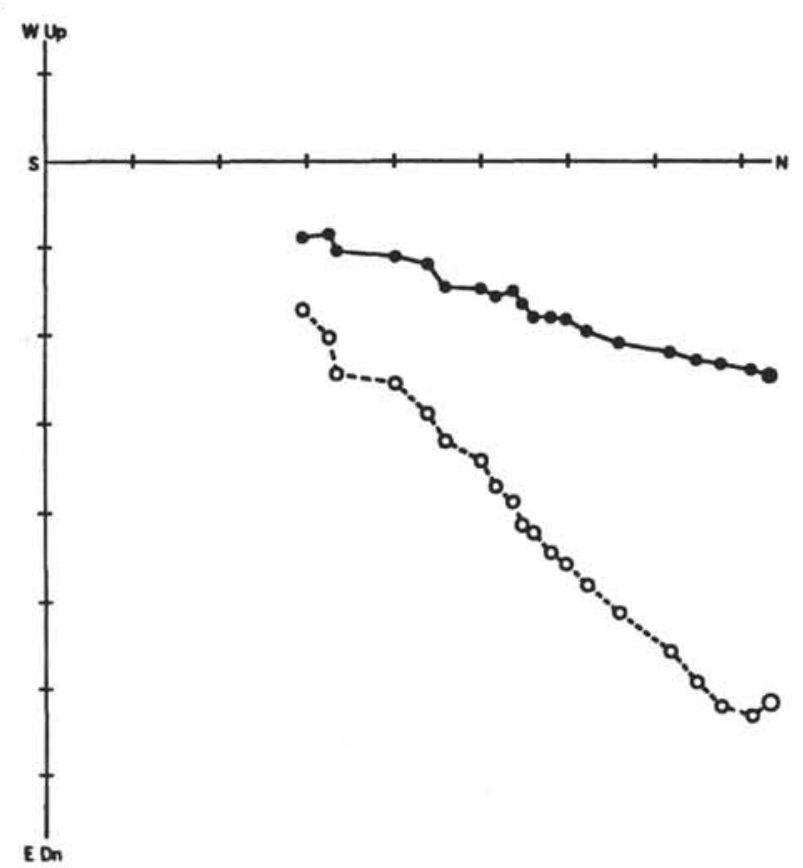

B

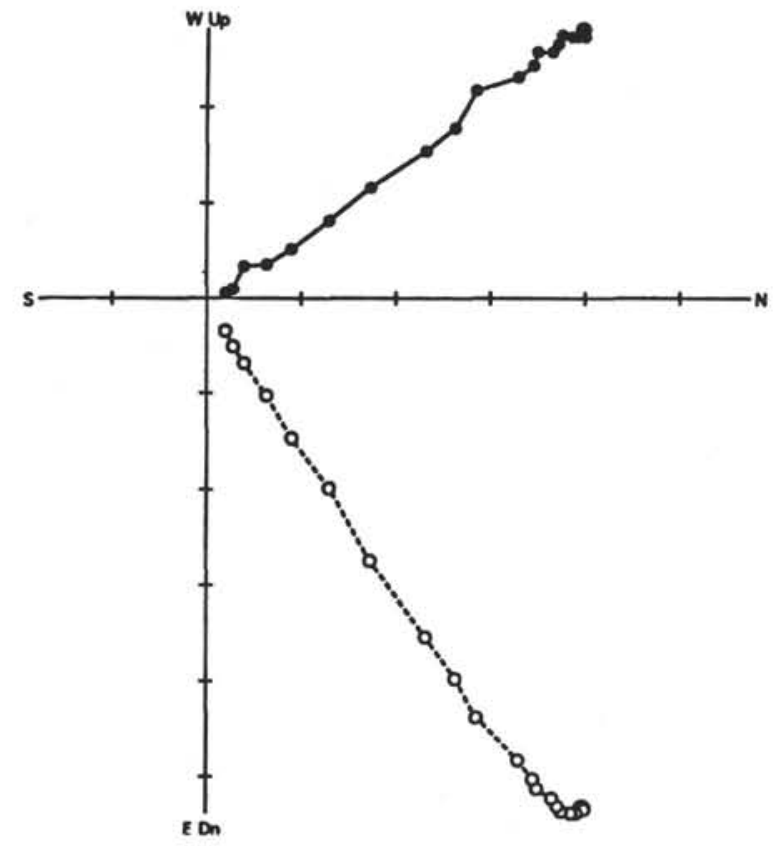

Figure 6. Zijderveld plots determined from AF demagnetization. A. Sample 147-894G-10R-1, 81-83 cm. B. Sample 147-894G-17R-1, 123-125 cm.

size is larger than that observed for single-domain magnetite, the gabbro samples are capable of carrying significant stable remanent magnetization.

Bulk coercivity $\left(H_{c}\right)$ measures the strength of the field that is required to drive the saturation magnetization of the sample to zero when measured in an applied field. It is generally used to determine the stability, or hardness, of the remanent magnetization carried by the sample. Values of $H_{c}$ are plotted vs. depth in Figure 7. The mean $H_{c}$ value for the Hole $894 \mathrm{G}$ gabbros is $10.5 \mathrm{mT}$. Although this indi- cates a moderate stability, this value is somewhat lower than expected based on the results of AF demagnetization. This difference probably reflects the response of different magnetic carriers to high and low field techniques. Specifically, AF demagnetization indicates that the carriers of the natural remanent magnetization are very hard, or stable, magnetically. Bulk coercivity, on the other hand, is a measure of the behavior of all of the grains in a high, or saturating, field. In this case, it appears that the bulk coercivity is not a perfect analog for the stability of natural remanent magnetization. Figure 8 shows a plot of the $\mathrm{J}_{r s} / \mathrm{J}_{s}$ values vs. $\mathrm{H}_{r c} / \mathrm{H}_{c}$ and demonstrates that the behavior of Hole $894 \mathrm{G}$ gabbro samples places them in the field described as pseudo-single domain.

\section{Magnetic Mineralogy}

Thermal demagnetization of 10 samples showed a sharp decrease in magnetization at $\sim 550^{\circ} \mathrm{C}$, indicating that nearly pure magnetite was the most important magnetic phase (Fig. 9). Measurements of magnetic susceptibility were made before and after thermal demagnetization and indicated that no signification change in magnetic mineralogy (e.g., oxidation) took place during the process of thermal demagnetization. Curie temperature $\left(T_{c u r i e}\right)$ measurements were made on two Leg 147 gabbros to constrain the composition of the magnetic minerals contained in these rocks more accurately. Values of $549^{\circ}$ and $545^{\circ}$ indicate that the dominant magnetic mineral is titanomagnetite with about $5 \%$ ulvospinel or other cations $\left(T_{\text {curie }}=582^{\circ} \mathrm{C}\right.$ for pure magnetite). Neither thermal demagnetization nor Curie temperature measurements indicated the presence of a magnetic phase other than magnetite in these samples. These observations are consistent with optical mineralogy studies (below), which indicate that the only significant magnetic phase is magnetite. These results also agree with those of Dunlop and Prevot (1982), Hall et al. (1989), and Pariso and Johnson (1993b), which showed that titanium-poor magnetite was the only significant magnetic phase in oceanic gabbros recovered from DSDP drilling, the Troodos Ophiolite, and ODP Hole 735B, respectively.

Polished thin sections of Hole $894 \mathrm{G}$ gabbros were examined in both transmitted and reflected light at magnifications ranging from $30 \times$ to $1200 \times$. Primary titanomagnetite and ilmenite were observed in most sections, although the amount of this phase varied from $<1 \%$ to a few percent. The primary titanomagnetite was commonly interstitial to the silicate phases or rounded and included within a silicate phase. Primary titanomagnetite grains consistently contained welldeveloped ilmenite lamellae, indicating that high-temperature $\left(>600^{\circ} \mathrm{C}\right)$ deuteric oxidation was an important process (e.g., Buddington and Lindsley, 1964; Haggerty, 1976). In comparison, evolved gabbroic rocks recovered from Hole 735B (iron-titanium oxide-rich gabbros and gabbronorites) also contained variable amounts of primary titanomagnetite, which experienced high-temperature deuteric oxidation. However, the more primitive olivine gabbros from Hole $735 \mathrm{~B}$ comprised most of the recovered rocks $(60 \%)$ but rarely contained primary titanomagnetite.

Secondary magnetite was common as rods along the cleavage planes of altered pyroxene, as small equant, unoriented grains within altered pyroxene, and as irregular grains in altered olivine. This alteration assemblage is very similar to that observed in gabbro samples from Hole 735B (Pariso and Johnson, 1993b). The primary difference in the nature of silicate alteration between the gabbro samples from these two localities is that the Hole $894 \mathrm{G}$ gabbros experienced pervasive greenschist facies metamorphism in addition to amphibolite facies metamorphism, whereas alteration minerals in the Hole $735 \mathrm{~B}$ gabbros were generally restricted to amphibolite and granulite facies. With the exception of two shear zones, greenschist facies metamorphic minerals were rare in the Hole 735B drill core. Nevertheless, the abundance and occurrence of secondary magnetite in Hole $894 \mathrm{G}$ gabbros is very similar to that observed in Hole $735 \mathrm{~B}$ gabbros. 


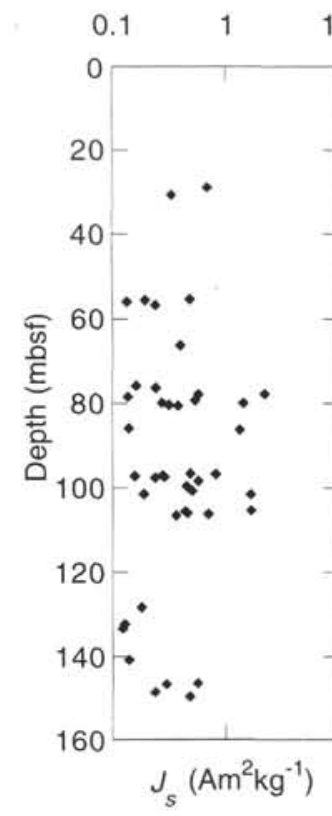

100
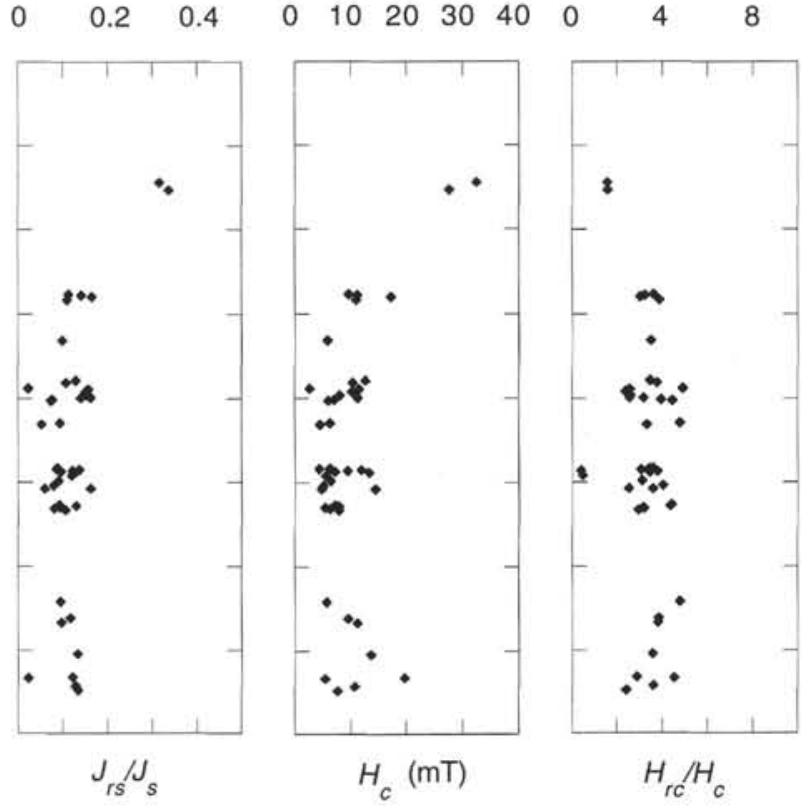

Figure 7. Saturation magnetization $\left(J_{s}\right)$, ratio of saturation magnetization to saturation remanence $\left(J_{r s} / J_{s}\right)$, bulk coercivity $\left(H_{c}\right)$, and ratio of coercivity or remanence and bulk coercivity $\left(H_{r c} / H_{c}\right)$ vs. depth for Hole $894 \mathrm{G}$ gabbro samples. Note that $J_{s}$ is plotted on a logarithmic scale.

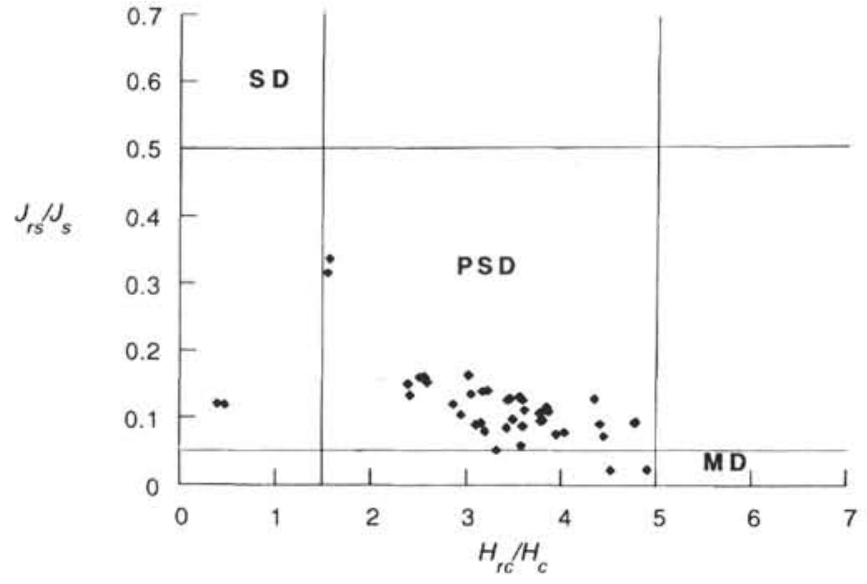

Figure 8. Values of $J_{r s} / J_{\mathrm{s}}$ plotted vs. $H_{r c} / H_{c}$ for Hole $894 \mathrm{G}$ gabbros. Boundaries for single domain (SD), pseudo-single domain (PSD), and multidomain (MD) magnetic behavior are after Day et al. (1977). Most of the Hole 894G gabbros fall within the region described as pseudo-single domain.

\section{DISCUSSION AND CONCLUSION}

One of the most interesting aspects of studying the Hole $894 \mathrm{G}$ drill core was the opportunity to explore the potential differences in crustal formation processes that operate at fast- vs. slow-spreading centers. In our study, we were particularly interested in the net effect of these geological processes on the magnetic properties of the crustal rocks. Our results demonstrate that both the paleomagnetic and rock magnetic properties of the Hole $894 \mathrm{G}$ gabbros fall within the range of those from gabbros recovered from slow-spreading ridges. In particular, the intensity of the remanent magnetization is moderately high (mean $J_{0}=2.3 \mathrm{~A} / \mathrm{m}$ ), the remanent magnetization is very stable, and hysteresis loop parameters indicate the effective magnetic grain size falls within the range described as pseudo-single domain. In other words, our results indicate that the Hole $894 \mathrm{G}$ gabbros, like those

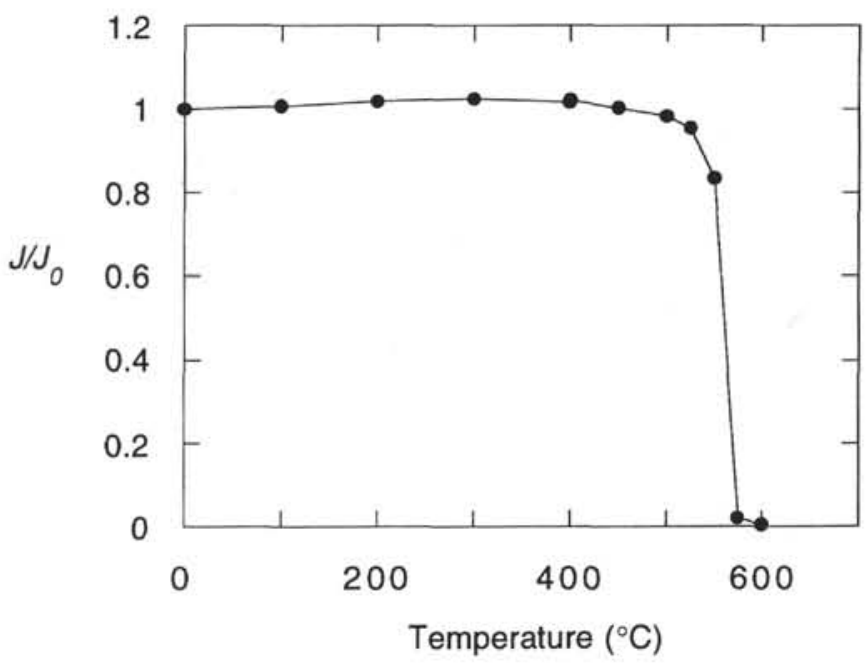

Figure 9. Thermal demagnetization of Sample 147-894G-17R-2, 65-67 cm.

from slow-spreading ridges, are capable of carrying significant stable remanent magnetization.

Both the highly fractured nature of the Hole $894 \mathrm{G}$ drill core and the drilling conditions indicated that the crustal section penetrated by Hole $894 \mathrm{G}$ consisted of highly fractured material. However, the range in $I_{\text {stable }}$ values is not high and compares well to that observed in Hole 735B (Pariso and Johnson, 1993a). Moreover, the range in $I_{\text {stable }}$ values for Hole $894 \mathrm{G}$ samples is much smaller than that observed in drill-core samples recovered from sections of extrusive basalt (e.g., Hole 504B; Furuta and Levi, 1983). Both the consistency of $I_{\text {stable }}$ values determined for Hole $894 \mathrm{G}$ samples and the strong evidence that remanent magnetization was acquired before uplift indicate that, although this crustal section may be highly fractured, the drilled section can accurately be described as a coherent (and reasonably intact) portion of lower oceanic crust. 
Magnetite in the Hole $894 \mathrm{G}$ gabbros is present as both a primary (i.e., igneous) and a secondary phase. The secondary magnetite formed as a result of the penetration of water into the crustal section at reasonably high temperatures. This result suggests that the remanent magnetization in this crustal section is a thermal remanent magnetization or a combination of thermal and chemical remanent magnetization, acquired soon after formation. In a general sense, our results suggest that gabbros like these are capable of making a significant contribution to a marine magnetic anomaly and suggests that it is important to consider the importance of Layer 3 when assessing possible source layers for marine magnetic anomalies. Unfortunately, because of its unusual tectonic environment, we cannot directly assess the ability of the Hole $894 \mathrm{G}$ gabbros to contribute to marine magnetic anomalies.

Coherent linear magnetic anomalies were not identified in the Hess Deep area. This is probably because of the tectonic and/or thermal disruption associated with the propagation of the Cocos-Nazca spreading center into crust formed at the East Pacific Rise. For example, the absence of an identifiable marine magnetic anomaly could be caused by a lack of a coherent magnetic direction in the bulk of the crustal section. Because we have only sampled a 150-m-thick section, it is possible that the general disruption of the crustal layers results in many different magnetic directions within a vertical section. In addition, we have no evidence that these gabbros acquired their remanent magnetization on the ridge axis. The steep inclination of the stable magnetic vector indicates that remanent magnetization of the Hole $894 \mathrm{G}$ section was acquired before uplift to its in situ position. Although it is likely that the Hole $894 \mathrm{G}$ section acquired remanent magnetization soon after formation, it is difficult to constrain the point between emplacement at the ridge and uplift to its current position when the magnetization was blocked in. Thus, based on the Hole $894 \mathrm{G}$ gabbros, we cannot determine whether Layer 3 rocks systematically record reversals of the Earth's magnetic field. Future drilling of Layer 3 rocks that are in their original position and have an associated, well-developed marine magnetic anomaly pattern may be the only means of addressing this long-standing problem.

\section{ACKNOWLEDGMENTS}

This work was supported by the JOI/USSAC fund for post-cruise science. We wish to thank Paul Johnson at the University of Washington and the Institute for Rock Magnetism (IRM) at the University of Minnesota for the use of their labs. The IRM is funded by the Keck Foundation, the National Science Foundation, and the University of Minnesota.

\section{REFERENCES}

Buddington, A.F., and Lindsley, D.H., 1964. Iron-titanium oxides minerals and synthetic equivalents. J. Petrol., 5:310-357.

Day, R., Fuller, M., and Schmidt, V.A., 1977. Hysteresis properties of titanomagnetites: grain-size and compositional dependence. Phys. Earth Planet. Inter., 13:260-267.

Dunlop, D.J., 1973. Superparamagnetic and single-domain threshold sizes in magnetite. J. Geophys. Res., 78:1780-1793.
Dunlop, D.J., and Prevot, M., 1982. Magnetic properties and opaque mineralogy of drilled submarine intrusive rocks. Geophys. J. R. Astron. Soc., 69:763-802.

Fox, P.J., and Opdyke, N.D., 1973. Geology of the oceanic crust: magnetic properties of oceanic rocks. J. Geophys. Res., 78:5139-5154.

Francheteau, J., Armijo, R., Cheminée, J.L., Hekinian, R., Lonsdale, P.F., and Blum, N., 1990. 1 Ma East Pacific Rise oceanic crust and uppermost mantle exposed by rifting in Hess Deep (equatorial Pacific Ocean). Earth Planet. Sci. Lett., 101:281-295.

Furuta, T., and Levi, S., 1983. Basement paleomagnetism of Hole 504B. In Cann, J.R., Langseth, M.G., Honnorez, J., Von Herzen, R.P., White, S.M., et al., Init. Repts. DSDP, 69: Washington (U.S. Govt. Printing Office), 697-703.

Haggerty, S.E., 1976. Oxidation of opaque mineral oxides in basalts. In Rumble, D., III (Ed.), Oxide Minerals. Short Course Notes-Mineral. Soc. Am., 3:101-140.

Hall, J.M., Fisher, B., Walls, C., Ward, T., Hall, L., Johnson, H.P., and Pariso, J., 1989. Magnetic properties, oxide petrography and alteration in the Cyprus Crustal Study Project Drill Hole CY-4 section though the lower sheeted complex and upper complex of the Troodos, Cyprus, ophiolite. In Gibson, I.L., Malpas, J., Robinson, P.T., and Xenophontos, C. (Eds.), Cyprus Crustal Study Project: Initial Report, Hole CY-4. Pap.-Geol. Surv. Can., 88-9:235-278.

Hekinian, R., Bideau, D., Francheteau, J., Cheminee, J.L., Armijo, R., Lonsdale, P., and Blum, N., 1993. Petrology of the East Pacific Rise crust and upper mantle exposed in the Hess Deep (eastern equatorial Pacific). $J$. Geophys. Res., 98:8069-8094.

Karson, J.A., Hurst, S.D., and Lonsdale, P.F., 1992. Tectonic rotations of dikes in fast-spread oceanic crust exposed near Hess Deep. Geology, 20:685-688.

Kent, D.V., Honnorez, B.M., Opdyke, N.D., and Fox, P.J., 1978. Magnetic properties of dredged oceanic gabbros and source of marine magnetic anomalies. Geophys. J. R. Astron. Soc., 55:513-537.

Kikawa, E., and Pariso, J.E., 1991. Magnetic properties of gabbros from Hole 735B, Southwest Indian Ridge. In Von Herzen, R.P., Robinson, P.T., et al., Proc. ODP, Sci. Results, 118: College Station, TX (Ocean Drilling Program), 285-307.

Lonsdale, P., 1988. Structural pattern of the Galapagos microplate and evolution of the Galapagos triple junction. J. Geophys. Res., 93:13551-13574.

McFadden, P.L., and McElhinny, M.W., 1984. A physical model for palaeosecular variation. Geophys. J. R. Astron. Soc., 78:809-830.

McFadden, P.L., and Reid, A.B., 1982. Analysis of paleomagnetic inclination data. Geophys. J. R. Astron. Soc., 69:307-319.

Pariso, J.E., and Johnson, H.P., 1993a. Do layer 3 rocks make a significant contribution to marine magnetic anomalies? In situ magnetization of gabbros at Ocean Drilling Program Hole 735B. J. Geophys. Res., 98:1603316052 .

_ 1993b. Do lower crustal rocks record reversals of the Earth's magnetic field? Magnetic petrology of oceanic gabbros from Ocean Drilling Program Hole 735B. J. Geophys. Res., 98:16013-16032.

Shipboard Scientific Party, 1993. Site 894. In Gillis, K., Mével, C., Allan, J., et al., Proc. ODP, Init. Repts., 147: College Station, TX (Ocean Drilling Program), 45-108.

Zijderveld, J.D.A., 1967. AC demagnetization of rocks: analysis of results. In Collinson, D.W., Creer, K.M., and Runcorn, S.K. (Eds.), Methods in Palaeomagnetism: New York (Elsevier), 254-286.

Date of initial receipt: 22 July 1994

Date of acceptance: 23 January 1995

Ms 147SR-023 